\title{
Phylogeographical particularity of the Mycobacterium tuberculosis Beijing family in South Korea based on international comparison with surrounding countries
}

Correspondence
Young Kil Park
ypark7@empal.com

Received 13 May 2010

Accepted 20 June 2010

\author{
Hee Yoon Kang, ${ }^{1}$ Takayuki Wada, ${ }^{2}$ Tomotada Iwamoto, ${ }^{3}$ Shinji Maeda, ${ }^{4}$ \\ Yoshiro Murase, ${ }^{4}$ Seiya Kato, ${ }^{4}$ Hee Jin Kim ${ }^{1}$ and Young Kil Park ${ }^{1}$ \\ ${ }^{1}$ Korean Institute of Tuberculosis, Korean National Tuberculosis Association, Seoul, \\ Republic of Korea \\ ${ }^{2}$ Department of Microbiology, Osaka City Institute of Public Health and Environmental Sciences, \\ Osaka, Japan \\ ${ }^{3}$ Department of Microbiology, Kobe Institute of Health, Kobe, Japan \\ ${ }^{4}$ Research Institute of Tuberculosis, Japan Anti-Tuberculosis Association, Tokyo, Japan
}

\begin{abstract}
To understand the domestic population structure of Mycobacterium tuberculosis clinical isolates in the Republic of Korea, we genotypically analysed 80 isolates obtained from various geographical origins in the country. Of these, 64 (80.0\%) isolates were identified as Beijing family strains. It is particularly interesting that their phylogenetic classification, based on the ancient/ modern separation and the presence/absence of the genomic region RD181, revealed a majority of the ancient (RD181+) subfamily in the population. The 15 loci of variable number of tandem repeat(s) of mycobacterial interspersed repetitive units (15-MIRU-VNTR) were also analysed.

Combination with the previous VNTR data reported from surrounding countries revealed that the topology of the minimum spanning tree was linked tightly not to the geographical origins of the patients but to the phylogenetic characteristics of the isolates. These results show that the phylogeographical distribution of the M. tuberculosis Beijing family around far-eastern Asia could be estimated using international accumulation and comparison of VNTR genotyping data.
\end{abstract}

\section{INTRODUCTION}

The Beijing family, a lineage of Mycobacterium tuberculosis, is well known for its highly endemic prevalence around East Asian countries and as a causative agent of tuberculosis (TB) (van Soolingen et al., 1995). In the Republic of Korea (South Korea), TB is still a major public health concern, with 34157 (70.3 per 100000$)$ registered new TB patients in 2008 and 2376 deaths attributable to TB in 2007 (Korea Centers for Disease Control \& Prevention, 2008). More than $70 \%$ of $M$. tuberculosis strains isolated from Korean pulmonary $\mathrm{TB}$ patients belong to the Beijing family. 'K strain' (Park et al., 2000), one of the Beijing family strains, has been reported as the cause of a severe outbreak of TB in South Korea (Kim et al., 2001). Although their phylogenetic position in the Beijing family

Abbreviations: MIRU-VNTR, variable number of tandem repeats of mycobacterial interspersed repetitive units; MST, minimum spanning tree; TB, tuberculosis.

Supplementary tables giving information on and genotypic profiles of the isolates analysed in this study are available with the online version of this paper. lineage has been unclear, Shamputa et al. (2010) reported recently that $\mathrm{K}$ strains show genetic diversity by some genotyping methods, even in isolates obtained from a single hospital.

The detailed phylogenetic variation of the Beijing family has been unveiled by various genetic markers such as single nucleotide polymorphisms, regions of difference and variable number of tandem repeat (VNTR) loci (Filliol et al., 2006; Tsolaki et al., 2005; Wada \& Iwamoto, 2009). A notable characteristic of the lineage is the insertion of IS6110 in a genomic region named NTF (Mokrousov et al., 2005; Plikaytis et al., 1994). This phylogenetic marker can classify the Beijing family into two distinct sublineages: ancient and modern. The modern Beijing sublineage has been more predominant than the ancient sublineage throughout the world, including countries surrounding South Korea (Bifani et al., 2002; Dou et al., 2008; Kremer et al., 2009; Mokrousov et al., 2005, 2006; van Soolingen \& Kremer, 2009). However, Wada et al. (2009) reported that the ancient Beijing sublineage has been observed to be exceptionally predominant in Japan. 
The goal of this study was to elucidate the phylogenetic distinctiveness and the genetic diversity of Beijing family strains around the Republic of Korea, including K family strains, for international comparison with strains from surrounding countries. For this purpose, we analysed a collection of $M$. tuberculosis isolates obtained from public health centres in the country using well-known phylogenetic markers and an international standard set of VNTR loci (Supply et al., 2006).

\section{METHODS}

Bacterial isolates. Eighty $M$. tuberculosis clinical isolates were analysed in this study. They were randomly selected from smearpositive and culture-positive pulmonary TB patients who were under 46 years of age with no epidemiological links during 2006. All patients were primary cases. Information such as geographical origin, age and sex of the patients, date of isolation and drug susceptibility for each of the 80 isolates is presented in Supplementary Table S1 in JMM Online. The geographical distribution of the population is presented in Fig. 1.

Identification of Beijing family strains and their phylogenetic subdivision. The Beijing family strains were defined by the deletion of RD207 in this study (Tsolaki et al., 2004). The deletion of RD207

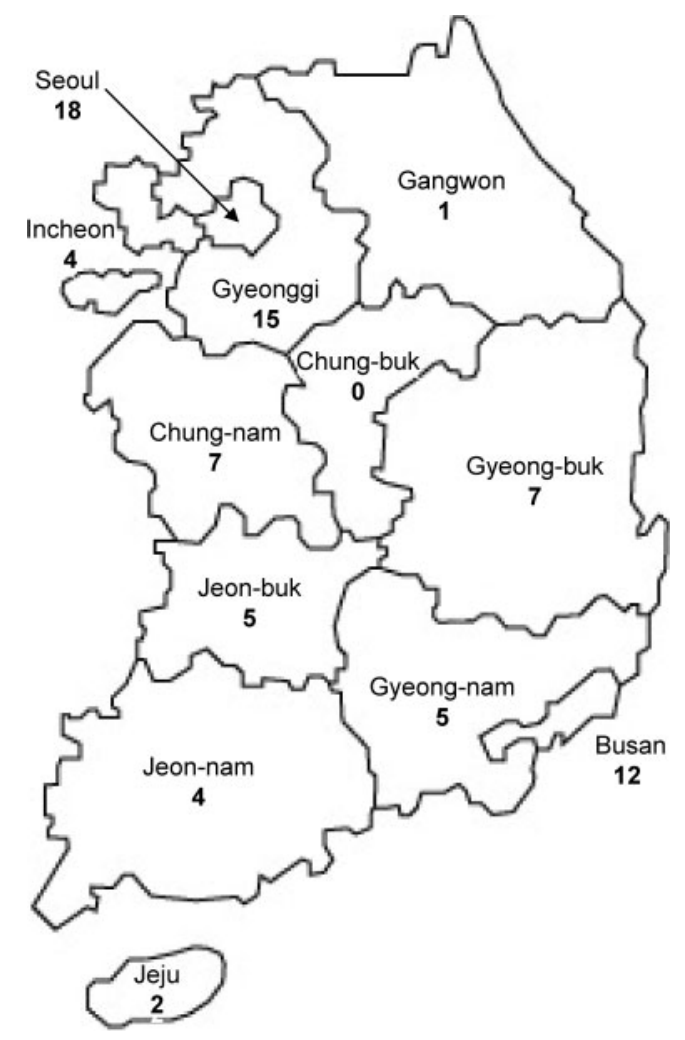

Fig. 1. Nationwide distribution of the M. tuberculosis strains used for this study. The $80 \mathrm{M}$. tuberculosis isolates were obtained from new pulmonary TB patients registered in public health centres across South Korea in 2006. corresponds with the absence of signals 1-34 in spacer oligonucleotide (spoligo) genotyping, which is the most standard definition of the lineage (van Soolingen et al., 1995). The presence or absence of RD207 was analysed using PCR according to a previous report (Warren et al., 2004).

The classification of the ancient and modern subfamilies of the 64 Beijing family isolates was determined according to a previous report (Wada et al., 2009). The presence or absence of RD181 was also verified as described previously (Tsolaki et al., 2004). The genomic deletion of RD181 has been considered to have occurred during evolution of the ancient Beijing sublineage (Tsolaki et al., 2004; Maeda et al., 2010). The sets of primer sequences were described in these previous reports. They were designed to detect insertions or deletions of regions by the difference in sizes of amplified DNA fragments.

IS6110 DNA fingerprinting. For all 80 isolates, DNA isolation and IS6110 RFLP typing were performed as described previously (Park et al., 2000). The IS6110 RFLP patterns were compared with UPGMA using the Dice coefficient to find RFLP clusters (GelCompar v. 5.1; Applied Maths). An RFLP cluster was defined by the identification of two or more isolates with identical band patterns. K family strains were identified according to the previously reported definition (Kim et al., 2001; Park et al., 2000).

VNTRs. The standard 15 VNTR loci for routine epidemiological discrimination (hereinafter, 15-MIRU-VNTR) were also analysed for all 80 isolates (Supply et al., 2006). Their copy number was calculated from their size and assigned according to the number of repeats for each locus, and in agreement with published allelic tables (Iwamoto et al., 2007). The accuracy of the size of amplified PCR fragments was confirmed using a capillary electrophoresis system (SV1210; Hitachi High Technologies). A VNTR cluster was defined by the identification of all 15 loci in two or more isolates.

Minimum spanning tree (MST). A MST was generated based on the 15-MIRU-VNTR types using software (Bionumerics v. 4.6; Applied Maths) for clustering analysis. We used the reconstruction rules as follows. A categorical coefficient was selected. The priority rule was set such that the type that had the highest number of single-locus variants would be linked first. Creation of hypothetical types was not allowed. The VNTR types of Beijing family strains from neighbouring countries were retrieved from previous reports (Jiao et al., 2008; Wada et al., 2009). All 15-MIRU-VNTR types published in these reports were incorporated into the construction of the MST tree.

\section{RESULTS}

\section{Phylogeographical specificity of the Beijing family in South Korea}

To elucidate the population structure of M. tuberculosis in South Korea, we identified $64(80.0 \%)$ Beijing family strains from 80 isolates obtained from various geographical origins (Fig. 1) and subdivided them into three phylogenetic sublineages [ancient (RD181+), ancient (RD181-) and modern]. A high proportion of the lineage is concordant with previous reports from South Korea and surrounding East Asian countries (Mokrousov et al., 2006; Park et al., 2000, 2005; van Soolingen et al., 1995; Yun et al., 2009). Phylogenetic subdivision of the 64 Beijing isolates revealed a higher proportion of the ancient Beijing sublineage (46 strains; $71.9 \%$ ) than the modern sublineage 
(18 strains; $28.1 \%)$. The ancient $(\mathrm{RD} 181+)$ sublineage, having diverged from the evolutionary process towards the modern Beijing sublineage before the deletion of RD181, was observed to be predominant (29 strains; $45.3 \%$ of the Beijing strains) in the population. This study identified $13 \mathrm{~K}$ family strains ( $16.3 \%$ of all; Fig. 2 ): all were found to belong to the ancient $(\mathrm{RD} 181+)$ sublineage.

\section{Genotypic diversity of isolates in South Korea}

We analysed all 80 isolates by 15-MIRU-VNTR to investigate the detailed genotypic diversity of the population. This genotyping method has been used as a standard discrimination tool for M. tuberculosis because of its high resolution among isolates from cosmopolitan origins (Supply et al., 2006). The VNTR profiles and other genotypic characteristics analysed in this study are combined and listed in Supplementary Table S2. The genotyping was able to classify our 80 isolates into 71 distinct genotypes. There were four clusters, which comprised $16(20.0 \%)$ isolates. The clustering rate was slightly higher than that of RFLP genotyping (10 isolates; $12.5 \%$ ), which was concordant with the previous reports on the Beijing family (Iwamoto et al., 2007; Yokoyama et al., 2007). Six of $13 \mathrm{~K}$ family strains belonging to two RFLP clusters were separated further into unique genotypes using 15-MIRU-VNTR (Fig. 3).

Recently, it was reported that cluster modelling of 15MIRU-VNTR genotypes of the M. tuberculosis Beijing strains was highly concordant with their phylogenetic subdivision into some sublineages (Wada \& Iwamoto, 2009). MST clustering analysis was performed for the 64 Beijing strains of our population (Fig. 4). The tree topology

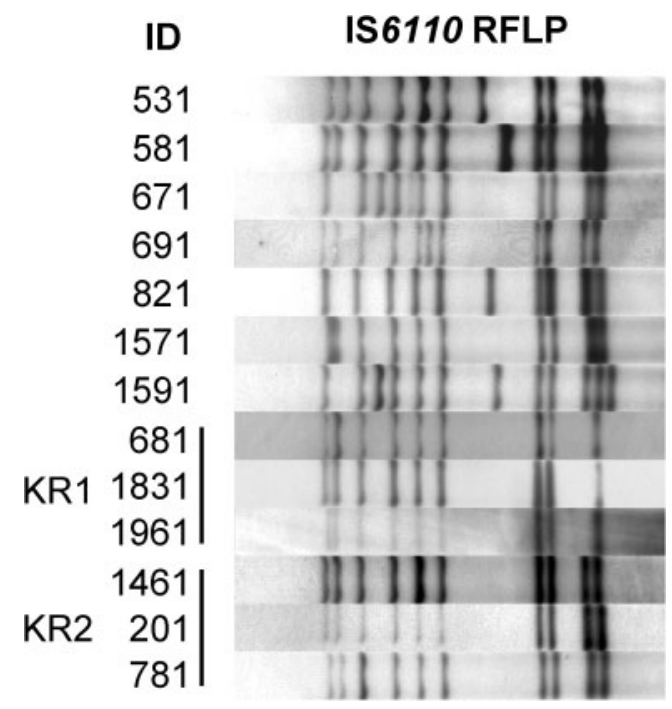

Fig. 2. IS6110 RFLP patterns of the $K$ family isolates identified in this study. Two clusters were detected in these 13 isolates (KR1, 681, 1831 and 1961; KR2, 1461, 201 and 781). was observed to be highly associated with the sublineage classification.

\section{Comparison of VNTR profiles with those of neighbouring countries}

The MST clustering of 15-MIRU-VNTR genotypes was sufficient to visualize the phylogenetic differences among the Beijing family successfully in our study (Fig. 4), which prompted us to make an international comparison of the 15-MIRU-VNTR types with those of strains from the neighbouring countries (China and Japan) reported previously. The 202 15-MIRU-VNTR types of Beijing strains from Japan (Wada et al., 2009) and the 64 types from Beijing City, China (Jiao et al., 2008), were combined with our current data to construct a mixed clustering tree using MST (Fig. 5). Results showed that three branches including types of strains from South Korea were readily apparent, two of which included mostly South Korean types (indicated by arrowheads). Both these branches comprised strains belonging to the ancient $(\mathrm{RD} 181+)$ sublineage, isolated in Japan and South Korea. A remaining branch included types from all three countries (indicated by an arrow). The results of the ancient/modern classification in the previous report by Wada et al. (2009) and this study showed that this branch was occupied by modern Beijing strains.

\section{DISCUSSION}

In general, the ancient Beijing sublineage has been considered to be an atypical Beijing genotype which has been only rarely observed (Bifani et al., 2002; Milan et al., 2004; Strauss et al., 2008). The sole exception has been the population study of Beijing family strains from Japan (Wada et al., 2009). In this study, we found that the ancient sublineage was predominant in South Korea. Contrary to the situation in Japan, it is unique that the ancient $(\mathrm{RD} 181+)$ sublineage was the most prevalent in our 80 isolates. This sublineage has been reported to be a minor component of the Beijing family population, even in Japan (Maeda et al., 2010). Therefore, our data also revealed the particularity of the population structure of the $M$. tuberculosis Beijing family in South Korea. These observations suggest that the distribution of the Beijing family sublineages is broadly variable in different regions. It still remains unclear whether the higher prevalence of the sublineages in certain areas has been caused by the difference of fitness or by occasional clonal expansion in the past.

$\mathrm{K}$ family strains, derivatives of the Beijing family, have been isolated frequently in both a population-based study and outbreaks of TB in South Korea (Kim et al., 2001; Shamputa et al., 2010). In this study, they were also detected with a high clustering rate $(46.2 \%)$ using RFLP genotyping (Fig. 2) despite no epidemiological link. They belonged to the ancient (RD181+) sublineage. Therefore, 


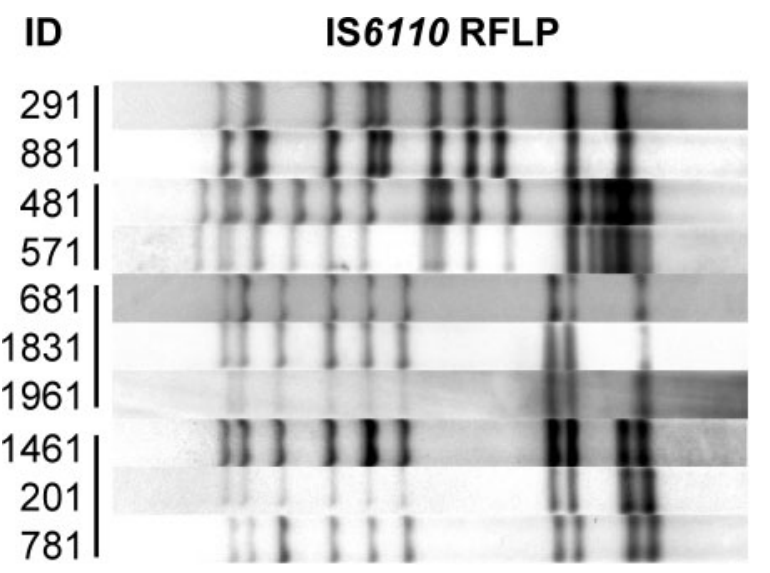

15-MIRU-VNTR

233353442722343

233353442722343

233753345834443

233753345834443

233742445943443

233752445843543

233842445943443

133742445943443

243742445953443

233742445943443

Fig. 3. 15-MIRU-VNTR profiles of 10 isolates belonging to four clusters by IS6110 RFLP genotyping. The order of the VNTR loci is: MIRU 04, MIRU 10, MIRU 16, MIRU 26, MIRU 31, MIRU 40, ETR A, ETR C, QUB-2163b, QUB-26, QUB-4156, Mtub04, Mtub21, Mtub30 and Mtub39. The alleles that differed from those of other isolates in the respective clusters are shaded.

the domestic prevalence of $\mathrm{K}$ family strains may be related to the predominance of the sublineage in the country. The 15-MIRU-VNTR genotyping was able to classify the RFLP clusters as single-locus to three-locus variants comprising the $\mathrm{K}$ family strains (Fig. 3). This result suggests that frequent isolation of $\mathrm{K}$ family strains in South Korea may be caused not by a recent expansion of a single strain but by endemic fixation in the past. The genetic diversity within the $\mathrm{K}$ family strains should be analysed in more detail to uncover the history of their prevalence.
Combining our data with the reported VNTR types of strains from the surrounding countries for MST clustering analysis revealed that the tree topology (the branch formation) was consistent with the phylogenetic classification of strains, irrespective of their origins (Fig. 5). The types of strains originating in China (Beijing) reported by Jiao et al. (2008) were concentrated in the branch of the modern Beijing sublineage of South Korea and Japan. Although their data did not include information on sublineages of strains, the result is consistent with those

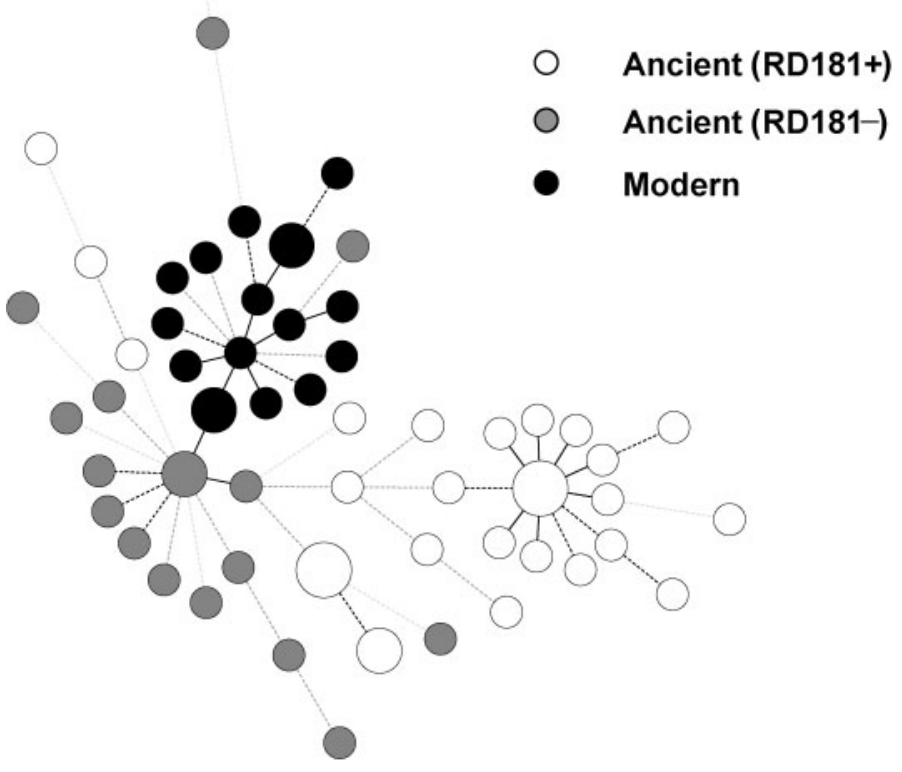

Fig. 4. Minimum spanning tree based on the 15-MIRU-VNTR genotypes of the $64 \mathrm{M}$. tuberculosis Beijing isolates. The 56 circles depicted correspond to the different types discriminated by the 15-MIRU-VNTR genotyping. Their sizes correspond to the number of isolates with a particular genotype. They were coloured according to the phylogenetic sublineages: ancient (RD181+), ancient (RD181-) and modern. Heavy lines connecting two types denote single-locus variants; thin lines connect double-locus variants; and dotted lines (black) connect triple-locus variants. The grey dotted lines represent the most likely connection between two types differing by more than three VNTR loci. 


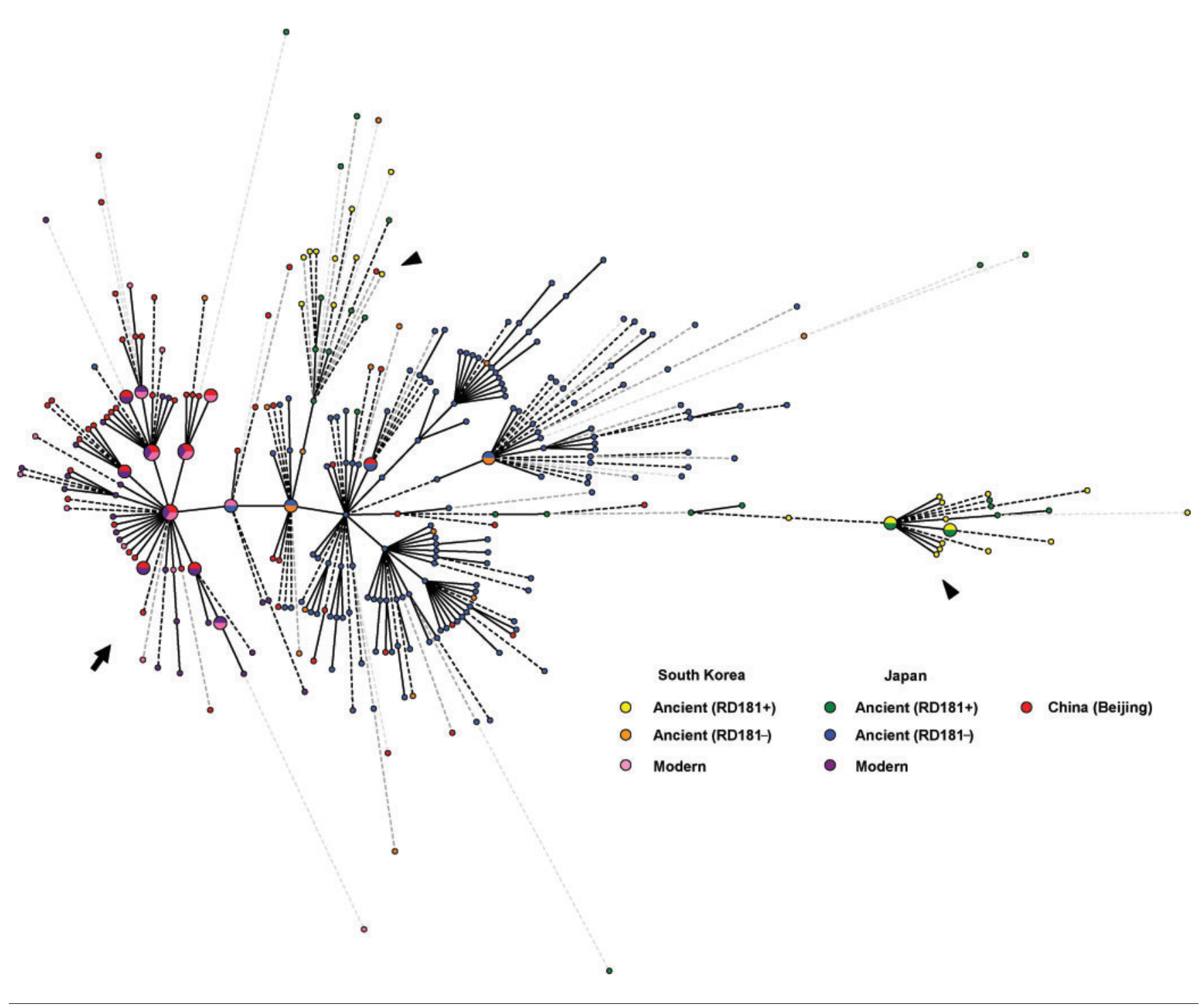

Fig. 5. Minimum spanning tree based on 15-MIRU-VNTR genotypes of the M. tuberculosis Beijing family from three East Asian countries. The 297 circles depicted correspond to the different types discriminated by the 15-MIRU-VNTR genotyping. The origin of each genotype is represented by different colours. The phylogenetic information of strains belonging to each genotype (from Japan and South Korea) is also indicated in colour. The designation of lines in the tree is the same as in Fig. 4. Two arrowheads point to clustering branches where genotypes originating from South Korea gather tightly. An arrow indicates a branch including genotypes of strains from all three countries.

of previous reports indicating that most of the isolated $M$. tuberculosis strains in the Beijing area (76-93\%) belonged to the modern Beijing sublineage (Mokrousov et al., 2006; van Soolingen \& Kremer, 2009). These results mean that similarity of 15-MIRU-VNTR types has been preserved in the ancient/modern sublineages of the Beijing family over the three East Asian countries. The phylogenetic validity of similar VNTR types must be verified in M. tuberculosis lineages because these genotypes are generally highly homoplasic. However, it is notable that similarity of VNTR types of the Beijing family has been observed based not on their geographical origins but in a phylogenetic manner. One merit of VNTR genotyping is its convenience for comparison of data from different countries (AllixBéguec et al., 2008; Mokrousov, 2008). Worldwide data accumulation of VNTR types of $M$. tuberculosis may shed light on the microevolution and genetic diversity of the species. Such global characterization of the phylogeographical distribution of the Beijing family may be useful in providing fundamental information about the ongoing worldwide expansion of the lineage.

\section{ACKNOWLEDGEMENTS}

This work was supported by a grant from the Ministry of Health, Labour and Welfare, Japan (Research on Emerging and Re-emerging 
Infectious Diseases, Health Sciences Research Grants) and the Korean Centers for Disease Control \& Prevention.

\section{REFERENCES}

Allix-Béguec, C., Harmsen, D., Weniger, T., Supply, P. \& Niemann, S. (2008). Evaluation and strategy for use of MIRU-VNTRplus, a multifunctional database for online analysis of genotyping data and phylogenetic identification of Mycobacterium tuberculosis complex isolates. J Clin Microbiol 46, 2692-2699.

Bifani, P. J., Mathema, B., Kurepina, N. E. \& Kreiswirth, B. N. (2002). Global dissemination of the Mycobacterium tuberculosis W-Beijing family strains. Trends Microbiol 10, 45-52.

Dou, H. Y., Tseng, F. C., Lin, C. W., Chang, J. R., Sun, J. R., Tsai, W. S., Lee, S. Y., Su, I. J. \& Lu, J. J. (2008). Molecular epidemiology and evolutionary genetics of Mycobacterium tuberculosis in Taipei. BMC Infect Dis 8, 170.

Filliol, I., Motiwala, A. S., Cavatore, M., Qi, W., Hazbon, M. H., Bobadilla del Valle, M., Fyfe, J., Garcia-Garcia, L., Rastogi, N. \& other authors (2006). Global phylogeny of Mycobacterium tuberculosis based on single nucleotide polymorphism (SNP) analysis: insights into tuberculosis evolution, phylogenetic accuracy of other DNA fingerprinting systems, and recommendations for a minimal standard SNP set. J Bacteriol 188, 759-772.

Iwamoto, T., Yoshida, S., Suzuki, K., Tomita, M., Fujiyama, R., Tanaka, N., Kawakami, Y. \& Ito, M. (2007). Hypervariable loci that enhance the discriminatory ability of newly proposed 15-loci and 24-loci variable-number tandem repeat typing method on $\mathrm{Myco-}$ bacterium tuberculosis strains predominated by the Beijing family. FEMS Microbiol Lett 270, 67-74.

Jiao, W. W., Mokrousov, I., Sun, G. Z., Guo, Y. J., Vyazovaya, A., Narvskaya, O. \& Shen, A. D. (2008). Evaluation of new variablenumber tandem-repeat systems for typing Mycobacterium tuberculosis with Beijing genotype isolates from Beijing, China. J Clin Microbiol 46, 1045-1049.

Kim, S. J., Bai, G. H., Lee, H., Kim, H. J., Lew, W. J., Park, Y. K. \& Kim, Y. (2001). Transmission of Mycobacterium tuberculosis among high school students in Korea. Int J Tuberc Lung Dis 5, 824-830.

Korea Centers for Disease Control \& Prevention (2008). Annual Report on the Notified Tuberculosis Patients in Korea. Seoul: Korea Centers for Disease Control \& Prevention.

Kremer, K., van der Werf, M. J., Au, B. K., Anh, D. D., Kam, K. M., van Doorn, H. R., Borgdorff, M. W. \& van Soolingen, D. (2009). Vaccineinduced immunity circumvented by typical Mycobacterium tuberculosis Beijing strains. Emerg Infect Dis 15, 335-339.

Maeda, S., Wada, T., Iwamoto, T., Murase, Y., Mitarai, S., Sugawara, I. \& Kato, S. (2010). The Beijing family Mycobacterium tuberculosis isolated from throughout Japan: phylogeny and genetic features. Int $J$ Tuberc Lung Dis 14, 1201-1204.

Milan, S. J., Hauge, K. A., Kurepina, N. E., Lofy, K. H., Goldberg, S. V., Narita, M., Nolan, C. M., McElroy, P. D., Kreiswirth, B. N. \& Cangelosi, G. A. (2004). Expanded geographical distribution of the $N$ family of Mycobacterium tuberculosis strains within the United States. J Clin Microbiol 42, 1064-1068.

Mokrousov, I. (2008). Genetic geography of Mycobacterium tuberculosis Beijing genotype: a multifacet mirror of human history? Infect Genet Evol 8, 777-785.

Mokrousov, I., Ly, H. M., Otten, T., Lan, N. N., Vyshnevskyi, B., Hoffner, S. \& Narvskaya, O. (2005). Origin and primary dispersal of the Mycobacterium tuberculosis Beijing genotype: clues from human phylogeography. Genome Res 15, 1357-1364.
Mokrousov, I., Jiao, W. W., Sun, G. Z., Liu, J. W., Valcheva, V., Li, M., Narvskaya, O. \& Shen, A. D. (2006). Evolution of drug resistance in different sublineages of Mycobacterium tuberculosis Beijing genotype. Antimicrob Agents Chemother 50, 2820-2823.

Park, Y. K., Bai, G. H. \& Kim, S. J. (2000). Restriction fragment length polymorphism analysis of Mycobacterium tuberculosis isolated from countries in the western pacific region. J Clin Microbiol 38, 191197.

Park, Y. K., Shin, S., Ryu, S., Cho, S. N., Koh, W. J., Kwon, O. J., Shim, Y. S., Lew, W. J. \& Bai, G. H. (2005). Comparison of drug resistance genotypes between Beijing and non-Beijing family strains of Mycobacterium tuberculosis in Korea. J Microbiol Methods 63, 165172.

Plikaytis, B. B., Marden, J. L., Crawford, J. T., Woodley, C. L., Butler, W. R. \& Shinnick, T. M. (1994). Multiplex PCR assay specific for the multidrug-resistant strain W of Mycobacterium tuberculosis. J Clin Microbiol 32, 1542-1546.

Shamputa, I. C., Lee, J., Allix-Beguec, C., Cho, E. J., Lee, J. I., Rajan, V., Lee, E. G., Min, J. H., Carroll, M. W. \& other authors (2010). Genetic diversity of Mycobacterium tuberculosis isolates from a tertiary care tuberculosis hospital in South Korea. J Clin Microbiol 48, 387394.

Strauss, O. J., Warren, R. M., Jordaan, A., Streicher, E. M., Hanekom, M., Falmer, A. A., Albert, H., Trollip, A., Hoosain, E. \& other authors (2008). Spread of a low-fitness drug-resistant Mycobacterium tuberculosis strain in a setting of high human immunodeficiency virus prevalence. J Clin Microbiol 46, 1514-1516.

Supply, P., Allix, C., Lesjean, S., Cardoso-Oelemann, M., RuschGerdes, S., Willery, E., Savine, E., de Haas, P., van Deutekom, H. \& other authors (2006). Proposal for standardization of optimized mycobacterial interspersed repetitive unit-variable-number tandem repeat typing of Mycobacterium tuberculosis. J Clin Microbiol 44, $4498-4510$.

Tsolaki, A. G., Hirsh, A. E., DeRiemer, K., Enciso, J. A., Wong, M. Z., Hannan, M., Goguet de la Salmoniere, Y. O., Aman, K., Kato-Maeda, M. \& other authors (2004). Functional and evolutionary genomics of Mycobacterium tuberculosis: insights from genomic deletions in 100 strains. Proc Natl Acad Sci U S A 101, 4865-4870.

Tsolaki, A. G., Gagneux, S., Pym, A. S., Goguet de la Salmoniere, Y. O., Kreiswirth, B. N., Van Soolingen, D. \& Small, P. M. (2005). Genomic deletions classify the Beijing/W strains as a distinct genetic lineage of Mycobacterium tuberculosis. J Clin Microbiol 43, 3185-3191.

van Soolingen, D. \& Kremer, K. (2009). Findings and ongoing research in the molecular epidemiology of tuberculosis. Kekkaku $\mathbf{8 4}$, 83-89.

van Soolingen, D., Qian, L., de Haas, P. E., Douglas, J. T., Traore, H., Portaels, F., Qing, H. Z., Enkhsaikan, D., Nymadawa, P. \& other authors (1995). Predominance of a single genotype of Mycobacterium tuberculosis in countries of east Asia. J Clin Microbiol 33, 32343238.

Wada, T. \& Iwamoto, T. (2009). Allelic diversity of variable number of tandem repeats provides phylogenetic clues regarding the Mycobacterium tuberculosis Beijing family. Infect Genet Evol 9, 921926.

Wada, T., Iwamoto, T. \& Maeda, S. (2009). Genetic diversity of the Mycobacterium tuberculosis Beijing family in East Asia revealed through refined population structure analysis. FEMS Microbiol Lett 291, 35-43.

Warren, R. M., Victor, T. C., Streicher, E. M., Richardson, M., Beyers, N., Gey van Pittius, N. C. \& van Helden, P. D. (2004). Patients with active tuberculosis often have different strains in the same sputum specimen. Am J Respir Crit Care Med 169, 610-614. 
Yokoyama, E., Kishida, K., Uchimura, M. \& Ichinohe, S. (2007). Improved differentiation of Mycobacterium tuberculosis strains, including many Beijing genotype strains, using a new combination of variable number of tandem repeats loci. Infect Genet Evol 7, 499-508.
Yun, K. W., Song, E. J., Choi, G. E., Hwang, I. K., Lee, E. Y. \& Chang, C. L. (2009). Strain typing of Mycobacterium tuberculosis isolates from Korea by mycobacterial interspersed repetitive units-variable number of tandem repeats. Korean J Lab Med 29, 314-319. 